\title{
Measuring the Proton Selectivity of Graphene Membranes
}

\author{
Michael I. Walker, ${ }^{1}$ Philipp Braeuninger-Weimer, ${ }^{2}$ Robert S. Weatherup, ${ }^{2}$ Stephan Hofmann, ${ }^{2}$ and Ulrich F. Keyser ${ }^{1}$ \\ ${ }^{1}$ Cavendish Laboratory, University of Cambridge, \\ J.J. Thomson Avenue, Cambridge CB3 OHE, United Kingdom \\ ${ }^{2}$ Department of Engineering, University of Cambridge, Cambridge CB3 OFA, United Kingdom
}

\begin{abstract}
By systematically studying the proton selectivity of free-standing graphene membranes in aqueous solutions we demonstrate that protons are transported by passing through defects. We study the current-voltage characteristics of single-layer graphene grown by chemical vapour deposition (CVD) when a concentration gradient of $\mathrm{HCl}$ exists across it. Our measurements can unambiguously determine that $\mathrm{H}^{+}$ions are responsible for the selective part of the ionic current. By comparing the observed reversal potentials with positive and negative controls we demonstrate that the as-grown graphene is only weakly selective for protons. We use atomic layer deposition to block most of the defects in our CVD graphene. Our results show that a reduction in defect size decreases the ionic current but increases proton selectivity.
\end{abstract}

The selective transport of ions across graphene membranes in aqueous solutions is a key issue for high profile applications such as water desalination and proton exchange membranes. A number of studies have reported simulations [1, 2], measurements of filtration through graphene membranes $[3-6]$ and the translocations of macromolecules [7-9]. Proton transport across graphene membranes is especially interesting as recent reports have suggested proton selective transport across intact graphene membranes [10]. However, there is little consensus regarding the mechanisms of transport and the appropriate experiments to conclude that a graphene membrane is proton selective.

Determining which ions cross a membrane is a problem that has been extensively studied in the field of ion channels. Selective transport of ions and macromolecules across biological membranes is critical to the operation of biological systems. Since the work of Hodgkin and Huxley in 1939 these have traditionally been investigated by electrophysiology techniques and single channel measurements [11]. These methods can be used to determine selective transport in the presence of leakage and multiple transport pathways.

An established and immediate measurement of ion selectivity is to set up a concentration gradient over the membrane as shown in Fig 1(a). This creates a driving force for diffusion for both the positive and negative ions. However if one of the ions can pass through the membrane more easily than the other then there will be a measurable net current flow across the membrane when the potential is zero, indicated by point $\mathrm{B}$ in the idealised current (I) - voltage (V) characteristic in Fig 1(b) [12]. This current can be stopped by applying an opposing electric field. The corresponding voltage is called the reversal potential and is illustrated as point A in Fig 1(b). The voltage required is predicted by the Nernst equation, but deviations from the expected value can be used to assess the importance of leakage currents [12].

Here we present experiments to study the selectivity of graphene membranes to protons in aqueous solutions. By

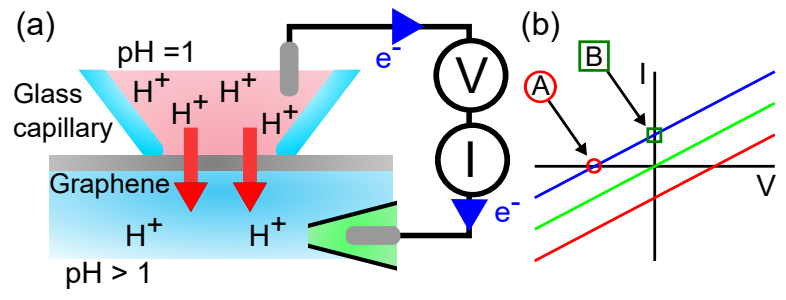

Figure 1. (a) A schematic of our experiment showing a glass nanocapillary sealed with a graphene membrane separating $\mathrm{HCl}$ solutions of different concentrations. $\mathrm{Ag} / \mathrm{AgCl}$ electrodes in the reservoir and the capillary connect the experiment to an amplifier which applies a voltage and measures the current. The arrows show the diffusion current of protons due to the concentration gradient. (b) An illustration of the expected changes in the I-V characteristic when a concentration gradient across a selective membrane drives a diffusion current at zero potential (B) and the reversal potential required to stop the current $(\mathrm{A})$.

systematically measuring the competition between $\mathrm{H}^{+}$ and $\mathrm{Cl}^{-}$ions to cross single layer graphene membranes we can distinguish which ion is carrying the current. This is critical because all measurements across a membrane will measure a leakage current. This current could be passing via the seal around the membrane, directly though the substrate or, importantly for graphene, be due to defects in the sample. To dissociate the selective effect from a leakage current we create a concentration difference and investigate the I-V characteristics. From the reversal potential we can determine the extent to which our CVD graphene membranes are selective to protons. To establish the effect of defects we block the pores using atomic layer deposition (ALD) and as a positive control present results using the proton selective membrane Nafion.

We seal graphene membranes on to the tips of pulled glass nanocapillaries with diameters of $\mathrm{d}=180 \mathrm{~nm}$ and $\mathrm{d}=2 \mu \mathrm{m}$ using the method developed in [13] and illustrated in Fig 1(a). The graphene adheres to the tips of the capillaries so that the solution in the reservoir can 
be exchanged for a different concentration. $\mathrm{Ag} / \mathrm{AgCl}$ electrodes in the capillary and reservoir carry the current which is measured using an Axopatch 200B amplifier (Molecular Devices) used to take I-V curve measurements. Graphene was grown by chemical vapour deposition in an Aixtron BM Pro (4 inch) reactor, using $25 \mu \mathrm{m}$ thick $\mathrm{Cu}$ foil (Alfa Aesar, 99.8\%) as the catalyst and $\mathrm{CH}_{4}$ (diluted in $\mathrm{Ar}$ and $\mathrm{H}_{2}$ ) as the precursor at $1050^{\circ} \mathrm{C}$ [14]. Our single-layer graphene significantly impedes the flow of current [13]. We keep the solution inside the capillaries at $0.1 \mathrm{M} \mathrm{HCl}$ and vary the concentration of the $\mathrm{HCl}$ solution in the reservoir. It is important to ensure the electrode potentials remain constant despite changing the concentration of the reservoir. To achieve this we use agarose coated electrodes made up in $0.1 \mathrm{M} \mathrm{KCl}$ solution (Fig 1(a)). Our negative control experiments, using bare capillaries, show that the solution can be exchanged without a significant current being induced when no membrane is present. As a positive control we will present results using the proton selective membrane Nafion [15]; a commercial proton exchange membrane. It is highly conductive to protons (cations) but blocks anions. The Nafion is $100 \mu \mathrm{m}$ thick and is contacted in the same way as the graphene.

Typical I-V curves for each material are shown in Fig 2. The I-V curves for a bare capillary show voltage offsets of less than $5 \mathrm{mV}$ which correspond to a small component of the current being selective due to the negative charge on the surface of the glass nanocapillaries. A fully selective membrane would cause a reversal potential of $58 \mathrm{mV}$ per unit $\mathrm{pH}$ difference, so this indicates that less than $10 \%$ of the current is selective. Most of the current flows through the centre of the capillary and is carried equally by the positive and negative ions. In contrast the Nafion membrane measurements show a clear shift in the voltage and current as the concentration of $\mathrm{HCl}$ in the reservoir changes (Fig 2(b)). Offsets of 15 to $20 \mathrm{mV}$ clearly indicate that a significant proportion of the current is due to protons. However, as the reversal potential does not shift by $58 \mathrm{mV}$ it is clear that there is also a significant leakage current. These results confirm that our experiment can detect and quantify selectivity in the presence of other ionic currents.

The I-V curves for an as grown graphene membrane show evidence for limited selectivity, Fig 2(c). The voltage offsets are of the order of $5-10 \mathrm{mV}$ which indicates that a small proportion of the current is proton selective. However, we find that the leakage currents dominate over any selective current for our CVD graphene membranes.

Given that defects in CVD graphene are known to influence ionic flow it is necessary to establish the extent to which defects affect the current. We therefore used an atomic layer deposition process (ALD) to block most of the defects. $\mathrm{Al}_{2} \mathrm{O}_{3}$ is deposited onto the $\mathrm{NaCl}$ supported graphene using a Cambridge Nanotech Savannah ALD system with a 20 cycle process at $200^{\circ} \mathrm{C}$, consisting of al- (a)

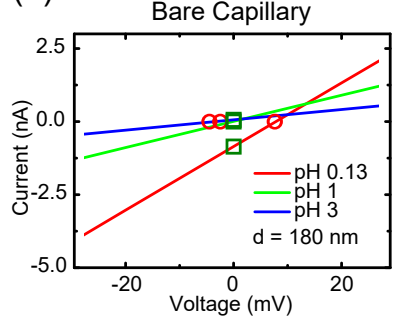

(c)

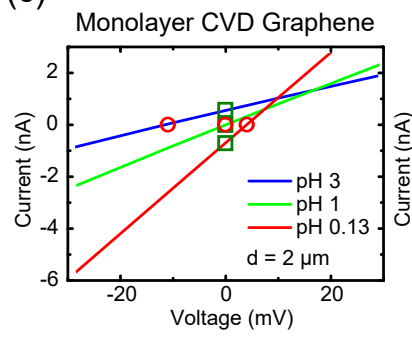

(b)

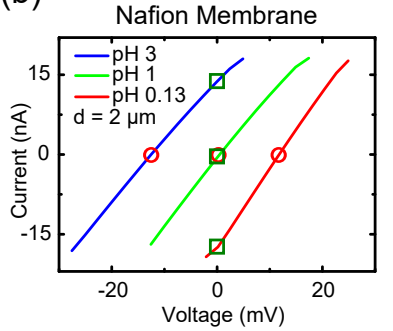

(d)

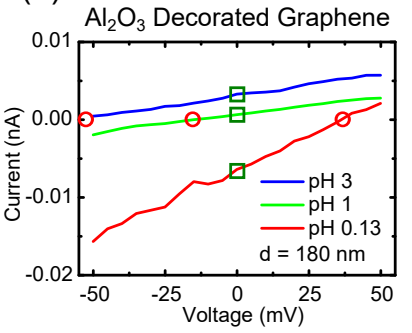

Figure 2. Typical I-V curves for different concentration differences. The concentration in the capillary is $0.1 \mathrm{M} \mathrm{HCl}$ and the $\mathrm{HCl}$ solution in the reservoir is indicated in the legends. The red circles indicate the reversal potential ("A") and the green squares indicate the diffusion driven current ("B"). We use capillaries with diameter $d=180 \mathrm{~nm}$ and $\mathrm{d}=2 \mu \mathrm{m}$ as indicated in the figure. (a) For a bare capillary (diameter $\mathrm{d}=180 \mathrm{~nm}$ ), the lines pass close to 0,0 indicating minimal selectivity associated with the charge on the glass. (b) The commercially available proton selective membrane Nafion (diameter $\mathrm{d}=2 \mu \mathrm{m})$. When there is a concentration difference across the capillary a current flows at $0 \mathrm{~V}$. This current can be stopped by applying the reversal potential. (c) Typical $\mathrm{I}-\mathrm{V}$ curves for an as grown monolayer graphene membrane, small voltage offsets indicate that there is weak evidence for selectivity (diameter $\mathrm{d}=2 \mu \mathrm{m}$ ). (d) Typical I-V curves for a graphene membrane decorated with $\mathrm{Al}_{2} \mathrm{O}_{3}$ (diameter $\mathrm{d}=180 \mathrm{~nm}$ ). Here the voltage offsets are similar to those for Nafion.

ternating pulses of trimethylaluminium and water both carried in a $\mathrm{N}_{2}(20 \mathrm{sccm})$ flow with $8 \mathrm{~s}$ purges between them $[16,17]$. This method typically yields a $2 \mathrm{~nm}$ thick film on $\mathrm{Si}$ with a native oxide. However, for this relatively high-temperature, water-based process the poor wetting of the $\mathrm{Al}_{2} \mathrm{O}_{3}$ on graphene is well documented, leading to preferential decoration at defects [16, 18-20]. It has been demonstrated that $\mathrm{Al}_{2} \mathrm{O}_{3}$ deposited by ALD reduces the ionic current [21] and our samples indeed showed significantly increased resistance (shown in supplementary information [22]). Typical I-V curves for $\mathrm{Al}_{2} \mathrm{O}_{3}$ decorated graphene are shown in Fig 2(d). The shape of these I-V curves more closely resembles those observed for Nafion than for either bare capillaries or as grown graphene. We measure reversal potentials of $15-25 \mathrm{mV}$, indicating that a significant proportion of the current is due to a proton flux, although the overall currents are the lowest of the three.

We can further quantify the selectivity of the mem- 
(a)

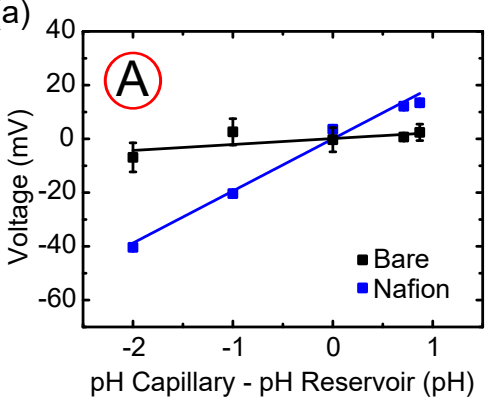

(b)

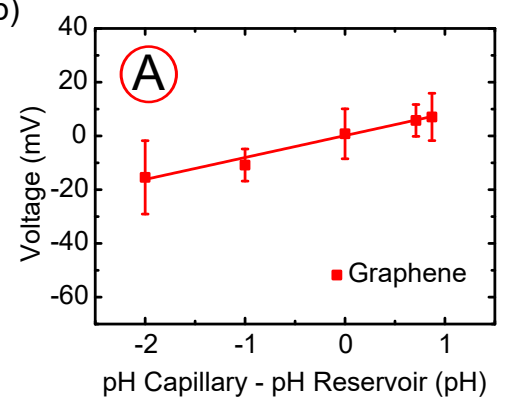

(c)

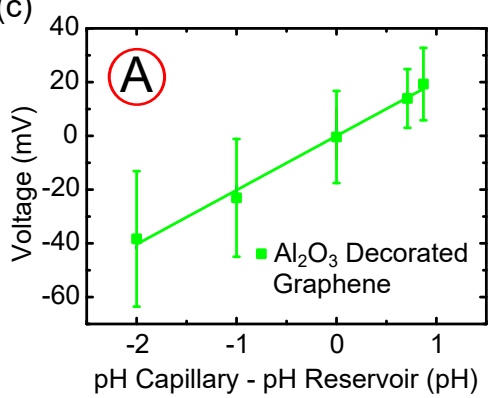

Figure 3. The voltage offsets plotted against the difference in capillary and reservoir pH for each of the four conditions (this corresponds to point "A" in Fig 1(b)). (a) The bare capillaries show a gradient of $2.2 \mathrm{mV} / \mathrm{pH}$ indicating the effect of negative charge on the surfaces of the glass nanocapillaries. The Nafion shows a $19.4 \mathrm{mV} / \mathrm{pH}$ offset indicating that the membrane is selective. (b) As grown graphene has a voltage offset of $8.1 \mathrm{mV} / \mathrm{pH}$. (c) $\mathrm{Al}_{2} \mathrm{O}_{3}$ decorated graphene shows an offset comparable with Nafion of $23 \mathrm{mV} / \mathrm{pH}$ indicating significant selectivity.

branes by analysing the reversal potentials (point $\mathrm{A}$ in Fig 1(a)). These are plotted in Fig 3(a-c) against the difference in $\mathrm{pH}$ of the solutions in the capillary and reservoir. From Fig 3 we observe that none of the fitted lines have a gradient of $58 \mathrm{mV} / \mathrm{pH}$ indicating that there is always a leakage current. The bare capillaries show evidence of very low selectivity with a gradient of $2 \mathrm{mV} / \mathrm{pH}$ whereas the Nafion is ten times more selective with a gradient of $20 \mathrm{mV} / \mathrm{pH}$ (Fig 3(a)). As grown graphene has a gradient of $8 \mathrm{mV} / \mathrm{pH}$, though the error bars are considerable (Fig 3(b)). This means that whilst there is some selectivity the bulk of current flow is due to non-selective leakage current. We do not observe a significant difference in selectivity between the results from $2 \mu \mathrm{m}$ and $180 \mathrm{~nm}$ capillaries. However, the graphene decorated with $\mathrm{Al}_{2} \mathrm{O}_{3}$ has a gradient of $23 \mathrm{mV} / \mathrm{pH}$ (Fig 3(c)) and exhibits proton selectivity comparable to Nafion.

The leakage conductivity for each membrane can be calculated from the ratio of the observed voltage per $\mathrm{pH}$ to the expected voltage per $\mathrm{pH}$ and the measured conductivity [23]. The leakage and selective pathways form a voltage divider relating the measured membrane voltage $V_{m}$, with the measured membrane conductance $G_{m}$, and the leakage conductance $G_{0}$, to the Nernstian potential $V[23]$.

$$
V_{m}=V \frac{G_{m}-G_{0}}{G_{m}}
$$

Using eq 1 we can estimate the selective proton conductivity for the as grown and $\mathrm{Al}_{2} \mathrm{O}_{3}$ decorated graphene, shown in Table I.

To consider where this selectivity arises from it is instructive to consider the current density at zero potential (point B in Fig 1(b)) plotted in Fig 4. For a selective membrane the concentration gradient will drive a current when the potential is zero. We see that both the as grown and decorated graphene show a current that scales with $\mathrm{pH}$. However for the $\mathrm{Al}_{2} \mathrm{O}_{3}$ decorated graphene it is much lower. We interpret this as current flowing through defects in as grown graphene. Adding $\mathrm{Al}_{2} \mathrm{O}_{3}$ blocks the defects decreasing both the selective and non selective components of current. This can also be seen in the values of the conductivities in Table I. The proton conductivity for as grown graphene is $1.3 \mathrm{nA} / \mu \mathrm{m}^{2}$ compared to $0.4 \mathrm{nA} / \mu \mathrm{m}^{2}$ for $\mathrm{Al}_{2} \mathrm{O}_{3}$ decorated graphene, despite the latter being three times more selective to protons.

We propose that the increase in selectivity is due to the defects decreasing in size and hence becoming more size selective for $\mathrm{H}^{+}$over $\mathrm{Cl}^{-}$. Blocking the defects decreases both the selective and non selective components of the conductivity through the graphene. This shows that the small amount of selectivity observed for the bare graphene membrane can also be attributed to selective defects. If the protons passed directly through the membrane as opposed to defects then we would not expect the proton current to decrease as significantly when the defects are blocked. In contrast to a recently published result [10], this indicates that proton transport across graphene membranes is via defects supporting the results in [24] which comes to a similar conclusion for graphene supported on a substrate.

An alternative analysis of our I-V characteristics can also be made by considering the Goldman Hodgkin Katz (GHK) equations [25]. These predict the shape of the I-V curve expected for a selective membrane [12]. See supplementary information for further details [22]. We only observed results which correspond to the GHK equations for the $\mathrm{Al}_{2} \mathrm{O}_{3}$ decorated graphene membrane (Fig 5). When the capillary is at a higher concentration than the reservoir, positive currents are larger indicating that protons can cross from the capillary into the reservoir. However when a negative voltage is applied the current is decreased since there are fewer protons in the reservoir to cross into the capillary, demonstrating that the $\mathrm{Cl}^{-}$ions in excess in the capillary are unable to cross the membrane and contribute to the current (blue line Fig 5). When 


\begin{tabular}{|c|c|c|c|c|}
\hline Membrane & $\begin{array}{c}\text { Selectivity } \\
(\mathrm{mV} / \mathrm{pH})\end{array}$ & $\begin{array}{l}\text { Conductivity } \\
\mathrm{nA} / \mu^{2}\end{array}$ & $\begin{array}{c}\text { Leakage } \\
\text { Conductivity } \\
\mathrm{nA} / \mu^{2}\end{array}$ & $\begin{array}{c}\text { Selective } \mathrm{H}^{+} \\
\text {Conductivity } \\
\mathrm{nA} / \mu \mathrm{m}^{2}\end{array}$ \\
\hline Bare Glass Nanopore & $2.2 \pm 0.56$ & 800 & 740 & 60 \\
\hline Nafion & $19.4 \pm 0.78$ & 18.7 & 12.5 & 6.2 \\
\hline As grown CVD graphene & $8.1 \pm 0.70$ & 16.4 & 15.1 & 1.3 \\
\hline $\mathrm{Al}_{2} \mathrm{O}_{3}$ decorated graphene & $23 \pm 1.6$ & 1.2 & 0.8 & 0.4 \\
\hline
\end{tabular}

Table I. Table of average selectivity in $\mathrm{mV} / \mathrm{pH}$ and the average conductivities of different samples broken down in to leakage and selective current.

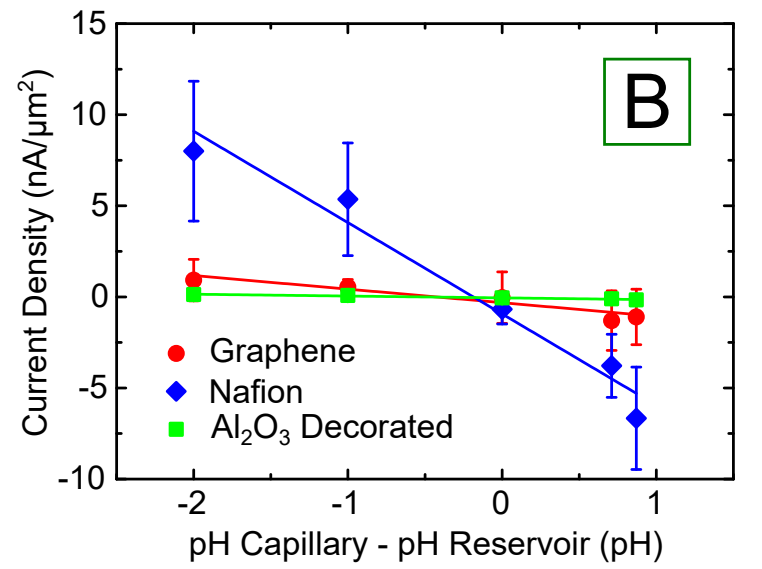

Figure 4. The current density at zero voltage plotted against the difference in capillary and reservoir $\mathrm{pH}$, for capillaries with diameter $\mathrm{d}=180 \mathrm{~nm}$ and $\mathrm{d}=2 \mu \mathrm{m}$ (this corresponds to point "B" in Fig 1(b)). This plot shows the concentration difference driven current when the amplifier in voltage track mode holds the voltage at $0 \mathrm{~V}$. All the samples show the current decreasing with increased $\mathrm{pH}$ in the reservoir as expected for selectivity. However the Nafion currents are much higher than those for graphene membranes. The lowest currents are across the $\mathrm{Al}_{2} \mathrm{O}_{3}$ sample. These results are presented separately in the supplementary information [22].

the concentration gradient is reversed the relative currents switch around so that the current due to a negative applied voltage is higher than the current for a positive applied voltage (red line Fig 5). The ratio between the permeability of the positive and negative ion indicates proton selectivity. When the reservoir is at $\mathrm{pH} 0.29$ we measure a ratio of 3.5 which would correspond to a reversal potential of $20 \mathrm{mV}$, consistent with the reversal potentials we have observed.

The high relative selectivity of the $\mathrm{Al}_{2} \mathrm{O}_{3}$ decorated graphene suggests it could be utilised as a selective membrane, for example in fuel cells. However, on the basis of our results we think there are significant challenges as the proton fluxes observed are much lower than for Nafion. The graphene membranes are either less selective or less permeable to protons than their commercial competitors.

Our results show that defects are critical for ionic transport and selectivity in our graphene membranes. Understanding the nature of these defects and how their

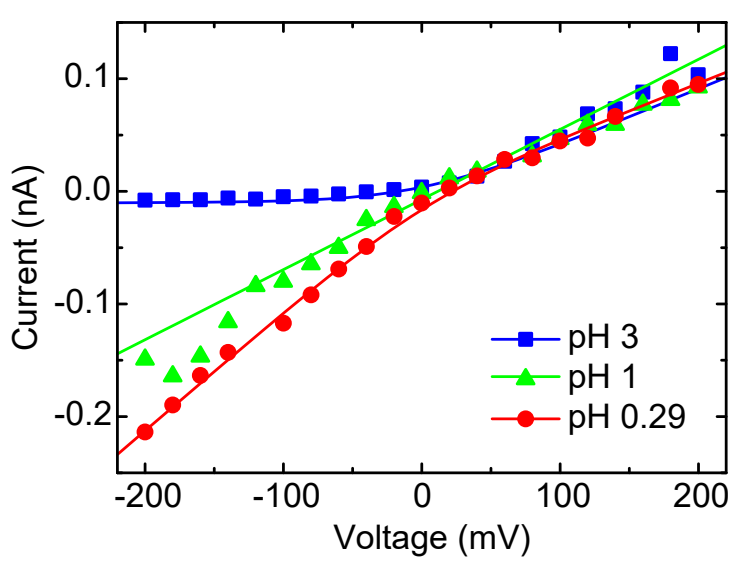

Figure 5. Plots of I-V curves for an $\mathrm{Al}_{2} \mathrm{O}_{3}$ decorated graphene membrane sealed across a $180 \mathrm{~nm}$ capillary. The capillary contains $0.1 \mathrm{M} \mathrm{HCl}$ and the concentration in the reservoir is exchanged. The lines show the fitted GHK equation, from which the permeability coefficients can be be determined.

size and chemistry influences their selectivity is a key challenge. We have shown that even a very small number of defects, or partially blocked defects can dominate ionic transport properties. Verifying that no defects exist in a graphene membrane is extremely challenging. The minimum defect density detectable in Raman spectroscopy is about $2 \times 10^{9} \mathrm{~cm}^{-2}$ hence up to 20 defects may exist per $1 \mu^{2}$ of graphene where Raman spectroscopy indicates defect free graphene [26-28]. Conversely TEM or STM have sufficient resolution to locally image individual defects but it is not practical to image the entirety of the graphene covering a $2 \mu \mathrm{m}$ pore.

We have measured the selectivity of Nafion and graphene membranes to $\mathrm{H}^{+}$by analysing the $\mathrm{I}-\mathrm{V}$ characteristics when there is a concentration difference across the membrane. Our results show that a leakage current does not prevent the use of established techniques to probe selectivity in aqueous solutions. By studying reversal potentials we have identified a small proton selective current through graphene membranes which can be reduced by blocking defects. We conclude that the proton selective current is through defects as opposed to across the graphene membrane.

The authors would like to thank C. Chimerel for use- 
ful discussions. This work was supported by the EPSRC Cambridge NanoDTC, EP/G037221/1 and EPSRC grant GRAPHTED, EP/K016636/1. RSW acknowledges a Research Fellowship from St. John's College, Cambridge and a Marie Skłodowska-Curie Individual Fellowship (Global) under grant ARTIST (no. 656870) from the European Union's Horizon 2020 research and innovation programme. All data accompanying this publication are directly available within the publication.

[1] K. Sint, B. Wang, P. Král, and P. Kra, J. Am. Chem. Soc. 130, 16448 (2008).

[2] Y. Kang, Z. Zhang, H. Shi, J. Zhang, L. Liang, Q. Wang, H. Agren, and Y. Tu, Nanoscale 6, 10666 (2014).

[3] S. P. Surwade, S. N. Smirnov, I. V. Vlassiouk, R. R. Unocic, G. M. Veith, S. Dai, and S. M. Mahurin, Nature Nanotechnology 10, 1 (2015).

[4] S. C. O'Hern, C. A. Stewart, M. S. H. Boutilier, J.-C. Idrobo, S. Bhaviripudi, S. K. Das, J. Kong, T. Laoui, M. Atieh, and R. Karnik, ACS Nano 6, 10130 (2012).

[5] S. C. O’Hern, M. S. H. Boutilier, J.-C. C. Idrobo, Y. Song, J. Kong, T. Laoui, M. Atieh, and R. Karnik, Nano Letters 14, 1234 (2014).

[6] S. P. Koenig, L. Wang, J. Pellegrino, and J. S. Bunch, Nature Nanotechnology 7, 728 (2012).

[7] S. Garaj, W. Hubbard, A. Reina, J. Kong, D. Branton, and J. A. Golovchenko, Nature 467, 190 (2010).

[8] G. F. Schneider, S. W. Kowalczyk, V. E. Calado, G. Pandraud, H. W. Zandbergen, L. M. K. Vandersypen, and C. Dekker, Nano Letters 10, 3163 (2010).

[9] C. A. Merchant, K. Healy, M. Wanunu, V. Ray, N. Peterman, J. Bartel, M. D. Fischbein, K. Venta, Z. Luo, A. T. C. Johnson, and M. Drndić, Nano Letters 10, 2915 (2010).

[10] S. Hu, M. Lozada-Hidalgo, F. C. Wang, A. Mishchenko, F. Schedin, R. R. Nair, E. W. Hill, D. W. Boukhvalov, M. I. Katsnelson, R. A. W. Dryfe, I. V. Grigorieva, H. A. $\mathrm{Wu}$, and A. K. Geim, Nature 516, 227 (2014).

[11] A. L. Hodgkin and A. F. Huxley, Nature 144, 710 (1939).

[12] B. Hille, Ion Channels of Excitable Membranes, 3rd ed. (Sinauer Associates, 2001) pp. $441-470$.

[13] M. I. Walker, R. S. Weatherup, N. A. W. Bell, S. Hof- mann, and U. F. Keyser, Applied Physics Letters 106, 023119 (2015).

[14] S. Hofmann, P. Braeuninger-Weimer, and R. S. Weatherup, The Journal of Physical Chemistry Letters 6, 2714 (2015).

[15] K. A. Mauritz and R. B. Moore, Chemical Reviews 104, 4535 (2004).

[16] B. Dlubak, P. R. Kidambi, R. S. Weatherup, S. Hofmann, and J. Robertson, Applied Physics Letters 100, 173113 (2012).

[17] R. S. Weatherup, C. Baehtz, B. Dlubak, B. C. Bayer, P. R. Kidambi, R. Blume, R. Schloegl, and S. Hofmann, Nano Letters 13, 4624 (2013).

[18] X. Wang, S. Tabakman, and H. Dai, J. Am. Chem. Soc. 130, 8152 (2008).

[19] J. A. Robinson, M. Labella, K. A. Trumbull, X. Weng, R. Cavelero, T. Daniels, Z. Hughes, M. Hollander, M. Fanton, and D. Snyder, ACS Nano 4, 2667 (2010).

[20] M. B. Martin, B. Dlubak, R. S. Weatherup, H. Yang, C. Deranlot, K. Bouzehouane, F. Petroff, A. Anane, S. Hofmann, J. Robertson, A. Fert, and P. Seneor, ACS Nano 8, 7890 (2014).

[21] S. C. O'Hern, D. Jang, S. Bose, J.-C. Idrobo, Y. Song, T. Laoui, J. Kong, and R. Karnik, Nano Letters 15, 3254 (2015).

[22] See supplementary material at [URL will be inserted by AIP] for examples of the graphene seals, detail of the current offsets and further information on the GHK equations.

[23] C. Chimerel, A. J. Murray, E. R. Oldewurtel, D. K. Summers, and U. F. Keyser, Chemphyschem 14, 417 (2013).

[24] J. L. Achtyl, R. R. Unocic, L. Xu, Y. Cai, M. Raju, W. Zhang, R. L. Sacci, I. V. Vlassiouk, P. F. Fulvio, P. Ganesh, D. J. Wesolowski, S. Dai, A. C. T. van Duin, M. Neurock, and F. M. Geiger, Nature Communications 6, 6539 (2015).

[25] D. E. Goldman, Journal of General Physiology 27, 37 (1943).

[26] L. G. Cançado, A. Jorio, E. H. M. Ferreira, F. Stavale, C. A. Achete, R. B. Capaz, M. V. O. Moutinho, A. Lombardo, T. S. Kulmala, and A. C. Ferrari, Nano Letters 11, 3190 (2011).

[27] A. C. Ferrari and D. M. Basko, Nature Nanotechnology 8, 235 (2013).

[28] M. Lucchese, F. Stavale, E. M. Ferreira, C. Vilani, M. Moutinho, R. B. Capaz, C. Achete, and A. Jorio, Carbon 48, 1592 (2010). 